\title{
Feature-specific border effects in the discrimination of letter-like forms
}

\author{
MURRAY J. WHITE \\ Victoria University of Wellington, Wellington, New Zealand
}

\begin{abstract}
Letter-like targets (a circle and a square) were presented in one of two fixed and cued visual field locations and were shown alone, flanked by a noise stimulus on the peripheral side (side of target farthest from fixation), on the central side, or on both sides simultaneously. The adjacent target and noise stimulus borders had similar featural properties (both curved or both straight lines) or dissimilar properties (one being a curved line and one a straight line). Each of 10 subjects made a go, no-go response only when his or her designated target appeared in a display. The results showed: (1) single targets were discriminated more accurately and more rapidly than were targets shown simultaneously with noise stimuli, (2) targets having dissimilar border relationships with noise items were discriminated more accurately than were targets having similar border relationships, (3) targets in central-noise displays were discriminated more accurately and rapidly than were targets in peripheral-noise displays, and (4) there was no interaction between border relationships and noise position. The principal result relating to target-noise border featural relationship was consistent with predictions derived from models which place the locus of noise effects at the stage of stimulus feature extraction. Aspects of the results were, however, seen to be consistent with both feature extraction and response competition conceptualizations.
\end{abstract}

Perhaps three of the most exhaustively researched questions about visual processing have to do with capacity limitations, attentional control, and lateral masking. The present paper is concerned with the last of these and, specifically, with the effects of targetnoise border featural similarity on target discrimination.

The main documented effects of lateral masking can be briefly summarized: (1) Target discrimination is impaired by the very presence of spatially adjacent noise items, targets accompanied by noise stimuli being discriminated less accurately than are targets shown with no accompanying noise stimuli (Bjork \& Murray, 1977; Eriksen \& Eriksen, 1974; Santee \& Egeth, 1980; Townsend \& Snodgrass, 1977). (2) A target shown with one or more noise items on its lateral side, farthest from the locus of fixation, its discriminated less well than is a target shown with noise items on its side nearest fixation (Banks, Bachrach, \& Larson, 1977; Banks, Larson, \& Prinzmetal, 1979; Chastain \& Lawson, 1979; Krumhansl, 1977; Krumhansl \& Thomas, 1977). (3) The noise-target featural relationship can have a profound effect on the rapidity and accuracy with which a target is discriminated. On this last point, however, the evidence is less clear-cut than it is with respect to the former two observations. Some researchers have shown that letter recognition

\footnotetext{
This research was supported by grants from the Internal Research Committee, Victoria University of Wellington, and from the University Grants Committee. The comments of Julie McKenzie and a consultant for Perception \& Psychophysics are gratefully acknowledged.
}

is impaired more when the target is surrounded by other letters than when it is surrounded by featurally dissimilar configurations, such as dot matrices (e.g., Estes, 1972). Others have found that recognition is poorer when a target letter is surrounded by featurally identical letters than when it is surrounded by featurally dissimilar letters (Bjork \& Murray, 1977), and yet others have reported directly opposite results (Eriksen \& Eriksen, 1974, 1979; Eriksen \& Schultz, 1979).

One established model of how noise stimuli might affect letter discrimination in visual processing is the interactive channels model of Estes $(1972,1974)$. In this, it is assumed that there are separate detectors for features such as curved and straight lines and that "as a result of instructions and experimental context, the input channels to detectors associated with the characters of the memory set involved in a particular experiment [e.g., the target set items] are put into a state of heightened excitability" (1972, p. 284). Further assumptions of this model are that the density of input channels decreases outwards from the fovea and that "excitation of any particular input channel exerts inhibitory effects on other channels going to the same or other feature detectors, the amount of inhibitory effect decreasing with distances in the visual field" (1972, p. 284).

According to Estes' model, discrimination of a target letter will be impaired more when shown in a display having spatially adjacent letters than when shown in a display having featurally dissimilar stimuli, and this impairment will be exacerbated, the greater the distance of the target letter outwards from fixation in the 
visual field. A modification to Estes' model has been proposed by Bjork and Murray (1977) in the light of their finding that the letter $\mathrm{B}$, for example, was discriminated less well when simultaneously presented with another B than when presented with an $\mathrm{R}$ or a $\mathrm{K}$. Bjork and Murray have proposed that "an active input channel exerts more inhibition on input channels leading to the same feature detector than to ones leading to other detectors"' (1977, p. 479). Further empirical support for Bjork and Murray's feature-specific inhibition model has been given by the experiments of Santee and Egeth (1980).

While the findings of Bjork and Murray and of Santee and Egeth place the majority of noise items effects at the stage of stimulus feature extraction, they are at variance with other findings which attribute the primary effects to processes occurring at a postperceptual response or decision level. In Eriksen and Eriksen's (1974) conceptualization, it is assumed that "there is a minimal channel size in terms of capacity for simultaneous processing with a capacity exceeding that required for identifying a single letter. Further, the unutilized capacity cannot be shut off and, if there are other letters or stimuli present, they will be processed simultaneously along with the target" (1974, p. 144). The visual processing model of Eriksen and Eriksen assumes that two or more stimuli can complete processing at the same time and begin evoking responses. However, because only one stimulus can be responded to at a time, some mechanism is required to determine which of the simultaneously processed items (e.g., target and noise item) should be responded to and which should not; that is, the effects of noise stimuli are presumed to create response competition between processed target and noise stimulus representations. This conclusion was, in turn, based on the finding that, with two letters ( $\mathrm{H}$ and $\mathrm{K}$ ) assigned to one levermovement response and two other letters ( $\mathrm{C}$ and $\mathrm{S}$ ) assigned to another lever-movement response, the target letter $\mathrm{H}$ was discriminated less rapidly and less accurately in displays having the form SSSHSSS than in displays having the form KKKHKKK. In the former, the processed noise $(\mathrm{S})$ and target $(\mathrm{H})$ items would have tended to elicit incompatible responses; in the latter, both items ( $\mathrm{H}$ and $\mathrm{K}$ ) would be compatible with a single response movement.

More recent studies by Eriksen and his associates have supported this finding and interpretation. Of particular importance, even when a lever movement is made compatible with both noise and target letters (e.g., $\mathrm{H}$ and $\mathrm{S}$ in displays having the forms $\mathrm{HHHHHHH}$ and SSSHSSS, or SSSSSSS and HHHSHHH), RTs are slower for targets surrounded by different letters. This finding, opposite to that predicted by Bjork and Murray's (1977) model, is handled by.positing competition effects among internal recognition responses: Response competition effects need not be localized at the level of overt response decision (Eriksen \& Eriksen, 1979; Eriksen \& Schultz, 1979).

The present experiment is specifically addressed to the question of the effects of target-noise border relationship on target discrimination and asks: Is target recognition impaired more when the adjacent target and noise items share similar features than when they have dissimilar features? In the experiments cited above, confusability or similarity has been defined in terms of shared or common features such as closed curves, vertical lines, and intersections (cf. Gibson, 1969). What this sort of analysis says is that the letter $\mathrm{O}$ is featurally more dissimilar to the letter $\mathrm{K}$ than it is to the letter $D$. The observation that $O$ (the target) is discriminated less accurately in a display of the form OD than in a display of the form OK can be attributed to target-noise differences in shared or common features. Some studies have, however, shown that the effects of interstimulus similarity on target discrimination might be specific to the nature of the adjacent target and noise border properties. In an experiment by Wolford and Hollingsworth (1974), it was found that the difference between recognition scores for the two end (rightmost) letters of multiple-letter displays was much greater when the penultimate end letter was asymmetrical (B, D, E) than when it was symmetrical (A, H, M). Again, Estes, Allmeyer, and Reder (1976) have found "differential effects of different mask characters on adjacent letters which have a vertical line or segment at the left side as distinguished from letters which do not"' (1976, p. 13). They noted that letters such as $B$ and $R$ were recognized less accurately than were letters such as $C$ and $S$ when the left side of a letter abutted a mask having the form \#. These findings highlight the possibility that the effects of target-noise featural similarily might be more specific than those conceptualized in the model of Bjork and Murray (1977). For example, while the target letter $O$ might be discriminated more accurately in the display $O K$ than in the display OD, in the OD type of display it might be discriminated better when the letters are in the lateral relationship OD than in the relationship DO; the borders of the former two letters are featurally more dissimilar than are the borders of the latter two.

If this notion is taken as a working hypothesis, it is nonetheless clear that for any potential effects due to border featural properties to be measured in a meaningful way, effects due to the lateral position of the noise stimulus need to be controlled. In displays having the forms OD and DO and centered on fixation, any superior recognition of the target $O$ in the display $O D$ might be due to the dissimilar target-noise border relationships and to the fact that $O$ is in a spatially different position in this display than it is in the display DO. If the displays were shown centered on some point in the left or right visual fields, potential border relationship effects could be confounded with effects due 
to the absolute position of the target $(\mathrm{O})$ from fixation. These considerations make it necessary to incorporate relative noise stimulus position as a factor in any study of the effects of target-noise border featural relationship on letter-form recognition (cf. Banks et al., 1977, 1979; Krumhansl, 1977; Krumhansl \& Thomas, 1977).

The present experiment is characterized by the following features: (1) The total stimulus set consists of two target stimuli (an outline of a circle and an outline of a square) and a single mask stimulus which has features common to both targets, being a composite of a half-circle and a half-square representing an elongated D. (2) The featural relationship between target and noise stimuli is defined in terms of whether the items share similar border features (two curved or two straight lines) or dissimilar features (one curved and one straight line). (3) The maximum display size is three items (one target flanked on both sides by a noise item), and the target is always shown in one of two predefined and cued visual field locations. In two blocks of trials, circle and square targets are shown without any accompanying noise stimuli; in three other blocks, a target is shown an equal number of times with a noise stimulus on the side that is farthest from fixation (peripherally), on the side that is nearest fixation (centrally), and on both sides simultaneously. When a target or target-plus-noise appears in the left visual field, the right visual field is blank, and vice versa. (4) The luminances of all display fields are matched and, except for the target and noise items, fixation marks, and target-location cues, are devoid of pattern. Each subject is required to make a simple go/no-go response only when his or her designated target (circle or square) is shown. Examples of the stimulus arrangements are shown in Figure 1.

\begin{tabular}{|c|c|c|c|c|}
\hline Target & $\begin{array}{l}\text { Target-Noise } \\
\text { relationship }\end{array}$ & $\begin{array}{c}\text { Noise } \\
\text { stimulus } \\
\text { position }\end{array}$ & s $\mid \frac{\text { Visua }}{\text { Left }}$ & $\frac{\text { field }}{\text { Right }}$ \\
\hline \multirow{2}{*}{ circle } & similar & $\begin{array}{c}P \\
C \\
P+C\end{array}$ & $\begin{array}{c}00 \\
00 \\
000\end{array}$ & $\begin{array}{l}00 \\
00 \\
000\end{array}$ \\
\hline & dissimilar & $\begin{array}{c}P \\
C \\
P+C\end{array}$ & $\begin{array}{l}\square 0 \\
O D \\
\square O\end{array}$ & $\begin{array}{l}O D \\
00 \\
0\end{array}$ \\
\hline \multirow{2}{*}{ square } & similar & $\begin{array}{c}P \\
C \\
P+C\end{array}$ & $\begin{array}{l}\square \square \\
\square D \\
\square \square D\end{array}$ & $\begin{aligned} & \square 0 \\
0 & \square \\
0 & \square\end{aligned}$ \\
\hline & dissimilar & $\begin{array}{c}P \\
C \\
P+C\end{array}$ & $\begin{array}{l}D \square \\
\square \square \\
\square \square \square\end{array}$ & $\begin{array}{l}\quad \square 0 \\
D \square \\
0 \square 0\end{array}$ \\
\hline
\end{tabular}

Figure 1. Representations of target-plus-noise stimulus displays. $P$, peripherally positioned noise stimulus; $C$, centrally positioned noise stimulus; $P+C$, peripherally and centrally positioned noise stimuli. (The target-to-noise distances are not drawn to scale.)

\section{METHOD}

\section{Stimulus and Apparatus}

Stimuli were presented binocularly in one field of a Gerbrands three-field tachistoscope in which the luminances of all fields were matched at $4.5 \mathrm{~cd} / \mathrm{m}^{2}$. Ambient room illumination was virtually zero. At the viewing distance, a circle target subtended an outside diameter of $.42 \mathrm{deg}$, a square target had a side length of $.42 \mathrm{deg}$, and a noise stimulus had a vertical length of $.42 \mathrm{deg}$ and a horizontal length of $.44 \mathrm{deg}$. The distance separating the outer adjacent edges of a target and a noise stimulus was $.14 \mathrm{deg}$, and the line stroke-width of all stimulus forms was $.04 \mathrm{deg}$. There were 32 nonoise displays and 72 target-plus-noise displays, and in both sets each target appeared equally often at each of two visual field positions, 2 deg to the left or 2 deg to the right of foveal fixation. In the target-plus-noise (noise) condition, equal numbers of all possible target-noise relationships and noise stimulus position combinations appeared at each visual field position.

\section{Procedure and Subjects}

The subjects first viewed an evenly illuminated field and, within 1,500 to 2,000 msec after the experimenter said "ready," a small fixation cross appeared in the middle of the field together with two pairs of vertically displaced bars, one bar directly above and one bar directly below the two points where a target could appear. These bars subtended vertical angular lengths of $.25 \mathrm{deg}$, with the nearest point of a bar $.52 \mathrm{deg}$ from the nearest edge of a target stimulus (assuming a temporal overlap of fields). Simultaneous with the onset of the fixation cross and cue markers, a $2,000-\mathrm{Hz}$ tone was sounded for $250 \mathrm{msec}$, warning the subject of an upcoming stimulus display. The fixation cross and cue markers were presented for $1,500 \mathrm{msec}$, and immediately on the offset of these, a stimulus display was shown for $50 \mathrm{msec}$. On the offset of this, the blank fieid was re-presented. The interval between successive trials (warning tone to warning tone) was $8 \mathrm{sec}$.

The subjects were tested individually, with each being instructed to rapidly depress two microswitches, one with the left index finger and one simultaneously with the right index finger, whenever one of the two target forms was shown located between the cue markers, and to not respond otherwise. This go/no-go, bimanual response procedure was employed for three reasons: (1) to minimize the RT variance (cf. Jonides \& Gleitman, 1976, Footnote 1), (2) to minimize any hemispheric response-initiation/stimulus-processing interaction effects which can result when stimuli are presented in the lateral visual fields and single-hand responses are called for (Geffen, Bradshaw, \& Nettleton, 1972; White \& White, 1975), and (3) to minimize effects due to incompatible overt response movements (Eriksen \& Schultz, 1979). In all cases, the depression of one switch was sufficient to stop the timer.

Five subjects were designated circle discriminators-responders and five, square discriminators-responders. Within each display set, the 32 no-noise and 72 noise displays were presented in random orders, given the constraint that equal numbers of all possible stimulus combinations appear in each successive run of 16 and 24 trials, respectively. The total sequence of trials went: one no-noise block ( 32 trials), three noise blocks ( $3 \times 72$ trials), and one no-noise block (32 trials). Eight female and two male graduate students with normal or corrected vision served as subjects.

\section{RESULTS}

\section{No-Noise Trials}

Targets in no-noise trials were discriminated more accurately and more rapidly than were targets in noise trials $(95.6 \%$ and $76.5 \%$, respectively; 466 and 700 msec, respectively). In the analysis of the no-noise accuracy data, there was no effect of type of target $[F(1,8)=3.1, p>.05]$, no visual field effect $(F<1)$, 
and no Target by Visual Field interaction. In the corresponding analysis of $\mathrm{RT}$ data, there was no visual field effect $(F<1)$ and no interaction between target and visual field $(F<1)$. There was, however, a small target effect with faster RTs for circle targets than for square targets (424 and $508 \mathrm{msec}$, respectively) $[F(1,8)$ $=6.29, \mathrm{MSe}=5,539.35, \mathrm{p}<.05]$.

\section{Noise Trials}

The findings of main interest with respect to the target-plus-noise (noise) trials are shown in Figure 2. In the analysis of the noise trials accuracy data, targets having dissimilar border relationships with noise stimuli were discriminated more accurately than were targets having similar border relationships $(80 \%$ and $73 \%$, respectively) $[\mathrm{F}(1,8)=7.92, \mathrm{MSe}=2.70, \mathrm{p}<.025]$. The noise stimulus position effect was also highly reliable $[\mathrm{F}(2,16)=27.77, \mathrm{MSe}=1.58, \mathrm{p}<.001]$, with target discrimination being impaired more by two surrounding noise elements $(68.9 \%$ ) than by either one peripheral noise element $(\mathbf{7 5 . 1 \% )}$ ) or one centrally situated noise element $(85.4 \%)$ and impaired more by a peripheral than by a central noise stimulus. (All these differences were significant at the .01 level by two-tailed $t$ test comparisons.) For the accuracy data analysis, visual field position, type of target, and all other interactions were insignificant at the .05 level.

In the analysis of noise trials RT data, the only reliable effect was that due to noise position $[F(2,16)=$ $17.84, \mathrm{MSe}=9,899.60, \mathrm{p}<.001]$. Targets surrounded by two noise elements were discriminated less rapidly

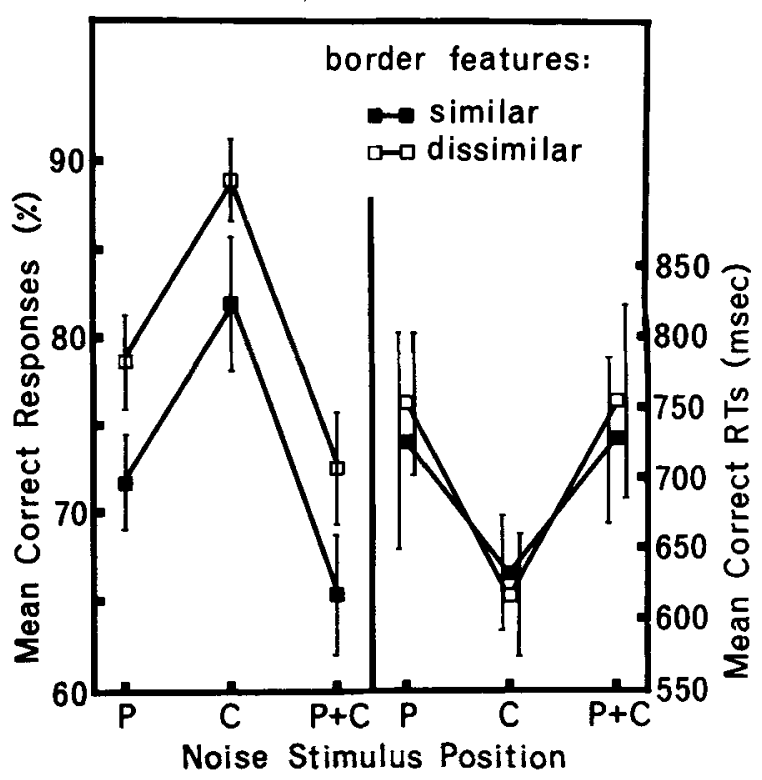

Figure 2. Mean correct discriminations and RTs for similar and dissimilar target-noise border relationships and noise stimulus positions. $P$, peripherally positioned noise stimulus; $C$, centrally positioned noise stimulus; $\mathbf{P}+\mathbf{C}$, peripherally and centrally positioned noise stimuli. Magnitudes of standard errors are shown by vertical bars.
(740 msec) than were targets flanked by one centrally positioned noise stimulus (624 msec) and targets having an adjacent peripheral noise stimulus were discriminated less rapidly $(738 \mathrm{msec})$ than were targets having an adjacent central noise stimulus. (These t-test comparisons were also significant at the .01 level.) Targets that had dissimilar border relationships with noise stimuli were discriminated as rapidly as were targets that had similar border relationships (707 and $694 \mathrm{msec}$, respectively). A suggestion of an interaction between target-noise border relationship and noise-stimulus position is apparent in Figure 2, but this interaction was less than reliable $(F<1)$, as might be expected from the overlap of the standard error distributions.

In the target-plus-noise trials, circle targets were again discriminated more rapidly than were square targets (628 and $773 \mathrm{msec}$, respectively), but the effect was not significant $(F<1)$. The result can be attributed to the fact that two subjects assigned (randomly) to the square-target response condition had mean RTs of 1,001 and 1,014 msec; the mean for all other eight subjects was $624 \mathrm{msec}$, with an SD of $86 \mathrm{msec}$. Of most importance here is the fact that in neither the accuracy analysis nor the RT analysis of the noise condition data did type of target interact with target-noise border relationship $[F<1$, and $F(1,8)=3.45, p>.10$, respectively, for accuracy and RTs]. In other words, while the absolute featural relationship between circle targets and circle border noise stimuli was different from that between square targets and straight-line border noise stimuli (the borders of the former two being composed of two curved lines facing different directions, with the borders of the latter two being composed of vertical lines-see Figure 1), the effect of circle border noise stimuli on circle targets was statistically equivalent to the effect of straight-line border noise stimuli on square targets.

\section{Practice Effects}

The effect of practice on performance was gauged by looking at the separate accuracy and $\mathrm{RT}$ results for the first and last blocks of no-noise trials and for the first and last blocks of noise trials. Mean accuracy in the first block of no-noise trials was $92.2 \%$ and in the last block, 98.1\% $[\mathrm{F}(1,8)=10.03, \mathrm{MSe}=1.80$, $\mathrm{p}<.01$; mean RTs were 473 and $461 \mathrm{msec}$, respectively $(F<1)$. There were no interactions between blocks (practice) and type of target. In order to give a sufficient number of data points for the noise-stimulus-trial comparisons, blocks of trials and type of target provided the factors in one analysis, and noise position, blocks of trials, and type of target were the factors in another. The mean accuracy rate for the first block of noise trials was $73.0 \%$, and for the last block was $77.1 \%$ $[\mathrm{F}(1,8)=4.14, \mathrm{MSe}=5.08, \mathrm{p}>.05]$. In neither of the accuracy analyses did blocks of trials interact with any other factor. The mean RTs for the first and last blocks of trials were the same, $671 \mathrm{msec}$, and no inter- 
actions between blocks, type of target, target-noise relationship, and noise position were found. In sum, while practice had the effect of increasing discrimination accuracy in no-noise trials, RTs remained stable. This finding might be attributed to the very simple response procedure used in the experiment, one which other researchers have found reduces RT variance (e.g., Jonides \& Gleitman, 1976). Most notably, practice had no differential effect on the performance of circle-target and square-target responders and, in the form of trial blocks, it did not interact with any of the principal experimental factors. (Parenthetically, it can be noted that there was no relationship between primary key depression for left- and right-finger responses, and visual field presentation, for RTs and accuracy.)

\section{Speed-Accuracy and False Alarms}

As a check on speed-accuracy tradeoffs, two tests were made of the noise trials data. In one, the pointbiserial correlation coefficient between RTs and discrimination errors was calculated for each subject, using the mean of the combined correct and false-alarm RTs as the cutoff for the dichotomous variable. These coefficients ranged from -.34 to +.05 , with a mean of -.09 . In the other, the mean correct RTs were compared with the mean false alarm RTs for all 10 subjects. This gave a $t(9)$ of $1.33(p>.05)$.

No formal tests were made of false-alarm RTs for the reasons that there were too few of these in the nonoise trials $(7.2 \%)$ and too many empty subject by treatment level cells in the noise trials (amounting to 28 of the 120 possible entries). At the most general level, it can be observed that the mean no-noise trials lalse-alarm RT (486 msec) did not differ appreciably from the mean correct RT (466 $\mathrm{msec})$, and that the mean noise trials false-alarm RT $(729 \mathrm{msec})$ was similar to the mean correct RT $(700 \mathrm{msec})$. (Because of the nature of the design, there were no RT misses. For example, while a display containing a circle target and not responded to by a circle subject could be recorded as a discrimination error, there was no corresponding error RT.)

\section{DISCUSSION}

A target having a noise stimulus on its side nearest fixation was discriminated much more accurately and rapidly than was a target having a noise stimulus on its side farthest from fixation. This result is consistent with one basic postulate of feature extraction models: The greater the distance stimuli are shown outwards from the locus of fixation in the visual field, the greater the competition for access to feature detectors due to decreases in the density of input channels (Bjork \& Murray, 1977; Estes, 1972). The result is, however, consistent with other conceptualizations. For example, Banks et al. $(1977,1979)$ have argued that as the midpoint of target-noise displays is moved farther out into the visual field, there is an increasing likelihood that the target and noise items will be perceived as featurally fused Gestalt configurations. (In the present experiment, the midpoint of a target-plus-peripheral noise display was $2.3 \mathrm{deg}$ from fixation, and the midpoint of a target-plus-central noise display was $1.7 \mathrm{deg}$ from fixation.)

Another explanation for the lateral asymmetry effect has been offered by Krumhansl (1977; Krumhansl \& Thomas, 1977). She proposes that feature perturbations occur during stimulus processing and that these occur more in a peripheral-to-foveal direction than in a fovealto-peripheral direction (cf. Wolford, 1975). In short, how one goes about interpreting lateral masking effects attributable to relative target-noise position still seems to be very much a matter of one's commitment to a particular theoretical position. A clear understanding of the locus of these effects has been rendered even more difficult by the results of Chastain and Lawson's (1979) experiment showing that a target form is recognized more accurately when accompanied by centrally positioned noise items than when accompanied by peripherally positioned noise stimuli, even when the midpoint of target-noise displays is at a constant distance from fixation from trial to trial. While, as Chastain and Lawson note, additional research is definitely implicated to nail down the locus of these pervasive noise position effects, it must be emphasized that, in the present experiments, noise position did not interact with the variable of primary interest, target-noise border relationship. The effect of similar and dissimilar border features was (statistically) independent of the relative position of the noise item with respect to the target.

The border relationship results, together with the target-alone vs. target-plus-noise conditions results, are in general agreement with other basic postulates of feature extraction models of visual processing. In particular, they appear to be consonant with the assumptions of Bjork and Murray's (1977) featurespecific inhibition model, that (1) "channels to the same feature detector inhibit each other more than they inhibit channels going to different detectors"' (1977, p. 482), and (2) "noise letters interfere with detection at the perceptual level to the extent that the target and noise letters in the same display utilize the same limitedcapacity feature detectors. This sharing of detectors is in turn a function of the similarity, proximity, and retinal location of the target and noise items" (1977, p. 483). Nevertheless, the present results suggest that a modification could be made to the second of these two assumptions, namely that the sharing of featurespecific detectors is a function of the similarity of the adjacent target and noise stimulus borders.

According to Bjork and Murray's argument, the target letter $\mathrm{D}$, for example, should be recognized more accurately in displays having the form DO than in displays having the form $D D$. The present results imply 
an opposite finding: Because the target-noise borders are more similar in the display DO than they are in the display DD, the letter $\mathrm{D}$ should be recognized more accurately in the latter type of display. The results lead to the conclusion that the interaction between feature input channels is, or can be, border-specific rather than letter- or form-specific. It has to be noted that the generality of the present findings has to be tested; the border-specific effects may not emerge so strongly when target and noise items are presented about the locus of fixation. And it has to be noted that a result such as that predicted above (better recognition of the letter $\mathrm{D}$ in the display DD than in the display DO) would be consistent with predictions derived from Eriksen and Schultz's (1979) continuous flow model, in which competition among internal recognition responses would be greater for the target $D$ in a DO display than for the target $\mathrm{D}$ in a DD display.

Why should both accuracy and RT measures reflect the effects of lateral noise position, yet effects due to target-noise border relationship be manifested only for correct discriminations? A tentative answer can be provided in terms of the conceptual distinction offered by Bjork and Murray (1977), that accuracy and response time measures are differentially sensitive to processes occurring at the perceptual and responsedecision levels of visual analysis. For the present results, the poorer discrimination accuracy for targets flanked by noise items having featurally similar borders and for targets having peripherally positioned noise items might be seen to reflect the effects of border feature-specific inhibition and the differential density of feature-specific channels in the visual field, these effects having their locus at the stage of percept formation. Significant RT results were observed only for the noise position variable and the longer RTs for correctly discriminated targets which were flanked by peripherally positioned noise stimuli might be taken as more indicative of processes occurring at a responsedecision level. In the experiment reported here, overt response competition effects attributable to the association of a particular response key or movement with particular target items were minimized by asking subjects to respond only when a single designated target appeared between the location markers. Nevertheless, it is reasonable to advance the hypothesis that some sort of competition among internal recognition responses accounted for the longer peripheral noise RTs if less definite information about target position was provided by the processed representations of targetplus-peripheral-noise displays than was provided by the representations of target-plus-central-noise displays. In other words, when clear representations of target and noise items were made available for an internal response decision, there was greater uncertainty as to which was the target and which the noise item for peripheral noise displays than for central noise displays (cf. Eriksen \& Schultz, 1979, p. 261; Estes et al., 1976, pp. 13-14).

The present experiment has provided some empirical evidence showing that target discrimination is impaired more when the border of a target is featurally similar to the border of a spatially proximate noise item than when the target-noise stimulus borders are dissimilar. This effect seems to be independent of the effects attributable to the relative lateral position of target and noise stimuli, even though noise position exerts a powerful influence on the accuracy and speed with which targets are discriminated. It has been contended that, in general, these results are more congruent with feature extraction (perceptual level) models than they are with models which attribute the effects of noise stimuli to competition among overt or covert recognition responses. However, it has to be conceded that aspects of these results are consistent with what would be predicted by both feature extraction and response decision types of models. This state of affairs seems to be consistent with other recently published studies (e.g., Eriksen \& Eriksen, 1979; Santee \& Egeth, 1980).

\section{REFERENCES}

Banks, W. P., Bachrach, K. M., \& Larson, D. W. Asymmetry of lateral interference in visual letter identification. Perception \& Psychophysics, 1977, 22, 232-240.

Banks, W. P., Larson, D. W., \& Prinzmetal, W. Asymmetry of visual interference. Perception \& Psychophysics, 1979, 25, 447-456.

Bjork, E. L., \& Murray, J. T. On the nature of input channels in visual processing. Psychological Review, 1977, 84, 472-484.

Chastain, G., \& Lawson, L. Identification asymmetry of parafoveal stimulus pairs. Perception \& Psychophysics, 1979, 26, 363-368.

ERIKSEN, B. A., \& ERIKSEN, C. W. Effects of noise letters upon the identification of a target letter in a nonsearch task. Perception \& Psychophysics, 1974, 16, 143-149.

Eriksen, C. W., \& Eriksen, B. A. Target redundancy in visual search: Do repetitions of the target within the display impair processing? Perception \& Psychophysics, 1979, 26, 195-205.

ERIKSEN, C. W., \& Schultz, D. W. Information processing in visual search: A continuous flow conception and experimental results. Perception \& Psychophysics, 1979, 25, 249-263.

Estes, W. K. Interactions of signal and background variables in visual processing. Perception \& Psychophysics, 1972, 12, 278-286.

Estes, W. K. Redundancy of noise elements and signals in visual detection of letters. Perception \& Psychophysics, 1974, 16, 53-60.

Estes, W. K., Allmeyer, D. H., \& Rede r, S. M. Serial position functions for letter identification at brief and extended exposure durations. Perception \& Psychophysics, 1976, 19, 1-15.

Geffen, G., Bradshaw, J. L., \& Netrleton, N. C. Hemispheric asymmetry: Verbal and spatial encoding of visual stimuli. Journal of Experimental Psychology, 1972, 95, 25-31.

GibSON, E. J. Principles of perceptual learning and development. New York: Appleton-Century-Crofts, 1969.

Jonides, J., \& Gleitman, H. Benefit of categorization in visual search: Target location without identification. Perception \& Psychophysics, 1976, 20, 289-298. 
Krumhansl. C. L. Naming and locating simultaneously and sequentially presented letters. Perception \& Psychophysics, 1977, 22, 293-302.

Krumhansl, C. L., \& Thomas, E. A. C. Effect of level of confusability on reporting letters from briefly presented visual displays. Perception \& Psychophysics, 1977, 21, 269-279.

SANTEE, J. L., \& EGETH, H. E. Interference in letter identification: A test of feature-specific inhibition. Perception \& Psychophysics, 1980, 27, 321-330.

Townsend, J. T., \& Snodgrass, J. G. Masking effects of similar and dissimilar letters: A test of the interactive channels model. Perceptual and Motor Skills, 1977, 44, 1219-1228.
White, M. J., \& White, K. G. Parallel-serial processing and hemispheric function. Neuropsychologia, 1975, 13, 377-381.

Wolford, G. Perturbation model for letter identification. Psychological Review, 1975, 82, 184-199.

Wolford, G., \& Hollingsworth, S. Lateral masking in visual information processing. Perception \& Psychophysics, 1974, 16, 315-320.

(Received for publication July 24, 1980; accepted revision received November 17,1980 .) 